\section{S109 ADAMTS13 PROTEIN LEVELS ARE DECREASED IN CHRONIC THROMBOEMBOLIC PULMONARY HYPERTENSION AND IMPLICATED IN ITS PATHOBIOLOGY}

${ }^{1} \mathrm{M}$ Newnham, ${ }^{2} \mathrm{~K}$ South, ${ }^{1} \mathrm{M}$ Bleda, ${ }^{3} \mathrm{~J}$ Cannon, ${ }^{1} \mathrm{~S}$ Gräf, ${ }^{1} \mathrm{C}$ Hadinnapola, ${ }^{3} \mathrm{~K}$ Sheares, ${ }^{3} \mathrm{D}$ Taboada, ${ }^{2} \mathrm{MR}$ Wilkins, ${ }^{2} \mathrm{~J}$ Wharton, ${ }^{3} \mathrm{~J}$ Pepke-Zaba, ${ }^{2} \mathrm{M}$ Laffan, ${ }^{2} \mathrm{DA}$ Lane, ${ }^{1} \mathrm{M}$ Toshner, ${ }^{1}$ NW Morrell. ${ }^{1}$ University of Cambridge, Cambridge, UK; ${ }^{2}$ Imperial College, London, UK;

${ }^{3}$ Papworth Hospital, Cambridge, UK

\subsection{6/thoraxjnl-2017-210983.115}

Introduction Chronic thromboembolic pulmonary hypertension (CTEPH) Results from failure of thrombus resolution following acute pulmonary embolism. Abnormalities in haemostasis are implicated in the pathobiology, including elevated levels of von Willebrand factor (VWF), which is normally regulated by ADAMTS13. Interim analysis of a genome-wide association study (GWAS) identified a significant association in CTEPH with the ADAMTS13 and ABO gene loci. We aimed to determine if ADAMTS13 protein levels are altered in CTEPH.

Methods ADAMTS13 and VWF plasma antigen levels were measured by ELISA in 208 individuals with CTEPH and compared to 68 healthy controls. Levels were also measured in subjects with chronic thromboembolic disease but without pulmonary hypertension (CTED), and other disease comparator groups summarised in figure 1. In $22 \mathrm{CTEPH}$ individuals ADAMTS13 and VWF levels were measured pre-operatively and at least 3 months post-pulmonary endarterectomy (PEA).

Results ADAMTS13 levels were decreased in CTEPH (median \pm IQR: $0.88 \pm 0.40 \mu \mathrm{g} / \mathrm{ml} ; \mathrm{p}=5.7 \times 10^{-09}$ ) and CTED $\left(0.83 \pm 0.22 \mu \mathrm{g} / \mathrm{ml} ; \mathrm{p}=2.1 \times 10^{-06}\right)$ patients compared to healthy controls $(1.15 \pm 0.30 \mu \mathrm{g} / \mathrm{ml})$ (figure 1). ADAMTS13 levels remained low in CTEPH patients following PEA (pre: 0.78 $\pm 0.27 \mu \mathrm{g} / \mathrm{ml}$ vs. post: $0.83 \pm 0.29 \mu \mathrm{g} / \mathrm{ml} ; \mathrm{p}=0.92$ ) even in those with normalised mean pulmonary arterial pressures $(<25 \mathrm{mmHg})$ after PEA. Furthermore, ADAMTS13 levels were lowest in the CTEPH and CTED groups when covariates (age, gender and batch) were included in multivariate rank regression models. VWF levels were increased in CTEPH $\left(16.7 \pm 15.2 \mu \mathrm{g} / \mathrm{ml} ; \mathrm{p}=4.0 \times 10^{-12}\right)$ and CTED $(17.0 \pm 10.1 \mu \mathrm{g} /$ $\left.\mathrm{ml} ; \mathrm{p}=3.9 \times 10^{-06}\right)$ compared to healthy controls $(8.5 \pm 8.8 \mu \mathrm{g} /$ $\mathrm{ml}$ ). There was no change post-PEA (pre: $22.2 \pm 17.3 \mu \mathrm{g} / \mathrm{ml}$ vs. post: $19.6 \pm 14.2 \mu \mathrm{g} / \mathrm{ml} ; \mathrm{p}=0.24)$.

Conclusions Plasma ADAMTS13 antigen levels are markedly decreased in CTEPH. This is not secondary to pulmonary hypertension, as demonstrated by the similarly low levels in CTED, and individuals with normal pulmonary artery pressures post-PEA. Thus, the VWF/ADAMTS13 axis is implicated in the underlying disease pathophysiology. Ongoing work will clarify if there is a causal link by defining whether genetic variation at the ADAMTS13 locus contributes to reduced ADAMTS13 protein levels and CTEPH.

\section{S110 HIF2A DELETION IN THE PULMONARY ENDOTHELIUM PREVENTS HYPOXIA-INDUCED PULMONARY HYPERTENSION}

AS Cowburn, A Crosby, D Macias-Gutierrez, M Southwood, C Branco, N Morrell, ER Chilvers, RS Johnson. University of Cambridge, Cambridge, UK

\subsection{6/thoraxjnl-2017-210983.116}

Pulmonary arterial hypertension is a progressive and irreversible disease that leads eventually to right heart failure and death. The pathogenesis of this condition involves proliferation of endothelial and smooth muscle cells resulting in vascular remodelling of the pulmonary arterioles. Several factors are implicated in the remodelling process driven by hypoxia including stabilisation of hypoxia-inducible transcription factors (HIFs), HIF1 $\alpha$ and HIF2 $\alpha$. Previous studies have shown that heterozygous deletions of HIF $1 \alpha$ or HIF $2 \alpha$ partially attenuate many of the remodelling process associated with the development of PAH. Consistent with these observations we have

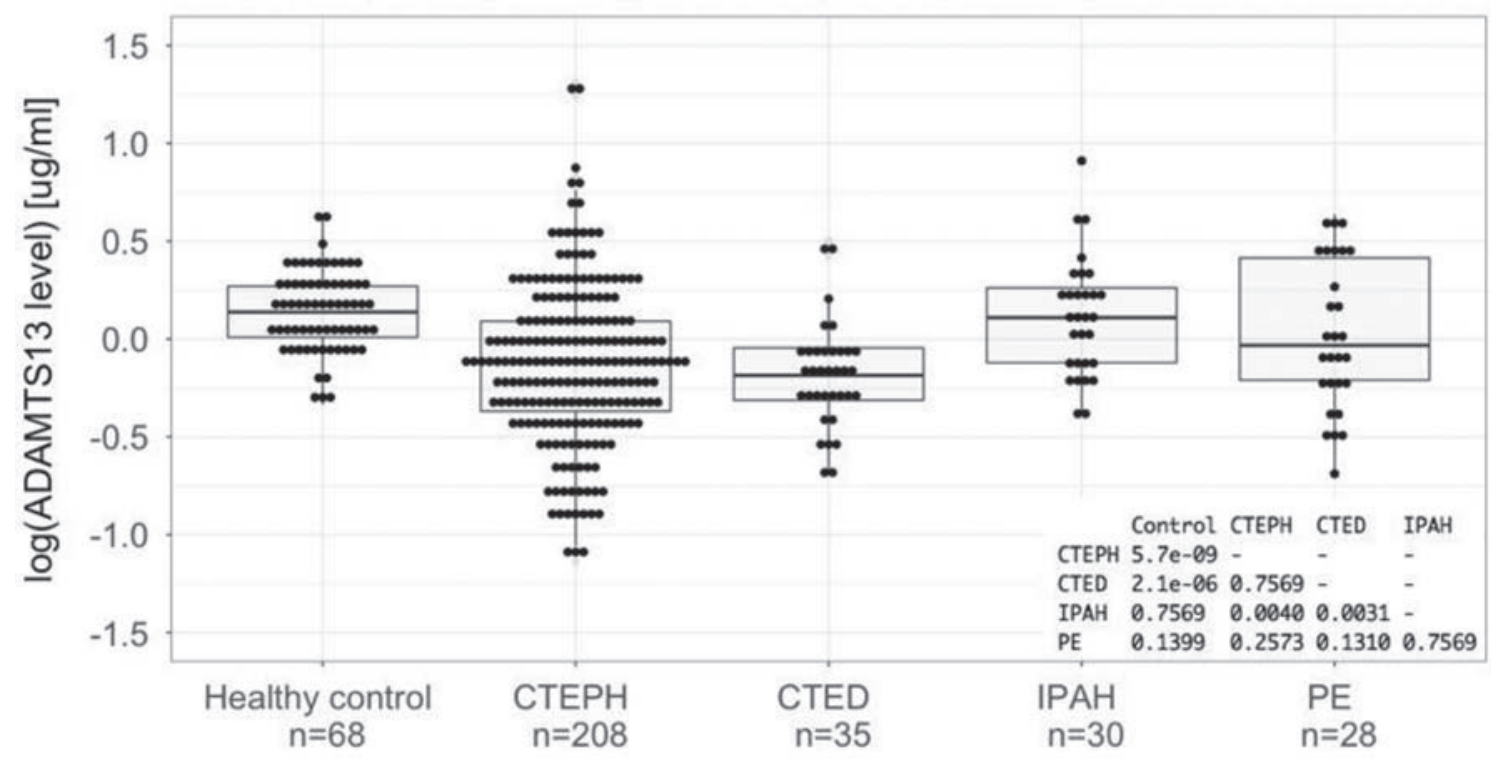

Diagnostic group

Abstract S109 Figure 1 ADAMTSI3 protein levels and diagnostic group. Log transformed ADAMTS13 antigen levels. Table of pairwise p-values displayed within figure (Dunns test). CTEPH, chronic thromboembolic pulmonary hypertension; CTED, chronic thromboembolic disease; IPAH, idiopathic pulmonary arterial hypertension; $\mathrm{PE}$, pulmonary embolism. 
found that pulmonary endothelial specific deletion of HIF2 $\alpha$, achieved using murine cre-loxp technologies (L1 or alk1-cre), offers protection against hypoxia-induced PAH. The rise in pulmonary artery pressure (PAP) normally observed following chronic hypoxic challenge was absent in mice with pulmonary endothelial HIF $2 \alpha$ deletion. The right ventricular systolic pressure of L1cre- HIF2 $\alpha$ mice post hypoxic challenge (26.17 $\pm 1.67 \mathrm{mmHg}, \mathrm{n}=7$ ) was not significantly different from untreated WT mice $(22.48 \pm 1.19 \mathrm{mmHg}, \mathrm{n}=9)$ and much lower than the hypertensive values seen in WT littermate controls $(41.91 \pm 1.88 \mathrm{mmHg}, \mathrm{n}=12, \mathrm{p}<0.0001)$ and L1cre-HIF1 $\alpha$ mice $(36.25 \pm 2.37 \mathrm{mmHg}, \mathrm{n}=7, \mathrm{p}<0.005)$. Only minimal remodelling was observed in lung sections from L1cre-HIF $2 \alpha$ mice reflecting the normal physiological PAPs following chronic hypoxia. We next questioned whether deletion of lung endothelial HIF $2 \alpha$ would be sufficient to reduce downstream arginase- 1 and -2 gene expression and in turn influence plasma nitrite/nitrate $\left(\mathrm{NO}_{(\mathrm{X})}\right)$ concentrations, which would be indicative of changes in nitric oxide homeostasis. The expression of both arginase- 1 and -2 were significantly reduced in hypoxia-conditioned whole lung samples from L1cre-HIF2 $\alpha$ mice relative to WT littermate controls. Plasma $\mathrm{NO}_{(\mathrm{X})}$ concentrations were also significantly elevated in the HIF $2 \alpha$ mutant mice when compared to plasma from WT control mice. These observations fit a model whereby reduced arginase- $1 / 2$ expression leads to increased availability of l-arginine, and in turn increased NO synthesis via NO synthases. These data offer new insights into the role of pulmonary endothelial HIF $2 \alpha$ in causing PAH, and offer new therapeutic opportunities for the treatment of this condition.

\section{S111 ALTERED NEUTROPHIL PHENOTYPES IN PULMONARY ARTERIAL HYPERTENSION}

${ }^{1} \mathrm{O}$ Dirir, ${ }^{2} \mathrm{KM}$ Lodge, ${ }^{1} \mathrm{~A}$ Creaser-Myers, ${ }^{1} \mathrm{~S}$ Walker, ${ }^{3} \mathrm{DG}$ Kiely, ${ }^{1} \mathrm{AM}$ Condliffe, ${ }^{1} \mathrm{~A}$ Lawrie, ${ }^{1}$ AAR Thompson. 'University of Sheffield, Sheffield, UK; ${ }^{2}$ University of Cambridge, Cambridge, UK; ${ }^{3}$ Sheffield Teaching Hospitals NHS Foundation Trust, Sheffield, UK

\subsection{6/thoraxjnl-2017-210983.117}

Introduction Evidence has implicated neutrophil elastase (NE), a proteolytic enzyme, as a key driver of the pulmonary vascular remodelling that underlies pulmonary arterial hypertension (PAH). Moreover, studies using animal models and explanted human lung tissue have demonstrated that inhibition of NE attenuates pulmonary hypertension. However, there has been little investigation into neutrophil function in $\mathrm{PAH}$ patients even though their azurophilic granules are the main physiological reservoir of NE. We investigated neutrophil phenotypes in patients with $\mathrm{PAH}$ versus healthy controls, with a focus on neutrophil degranulation.

Methods Neutrophils were isolated from venous blood of PAH patients and healthy controls (HC) and treated with LPS; viability was assessed at 20 hours by morphology. Cell surface receptor expression was determined by flow cytometry. To evaluate degranulation, neutrophils were treated with priming agents, platelet activating factor (PAF, $1 \mu \mathrm{M}$ ) or tumour necrosis factor- $\alpha$ (TNF, $20 \mathrm{ng} / \mathrm{ml}$ ), and subsequently stimulated with N-formylmethionyl-leucyl-phenylalanine (fMLP; $100 \mathrm{nM}$ ). NE release was measured by ELISA and released NE activity and myeloperoxidase (MPO) activity were determined by fluorogenic (Enzchek) and colorimetric (o-dianisidine oxidation) assays respectively.

Results Neutrophil apoptosis 20 hours following stimulation with LPS was significantly lower in PAH $(25.4 \%+/-2.2$, $\mathrm{n}=12)$ versus $\mathrm{HC}$ samples $(44.9 \%+/-4.7, \mathrm{n}=9), \mathrm{p}<0.001$. There were no differences in TLR2 or TLR4 expression between $\mathrm{PAH}$ and $\mathrm{HC}$ neutrophils. $\mathrm{PAH}$ neutrophils released greater amounts of NE following stimulation (e.g., TNF- $\alpha$ priming: PAH $675.2 \mathrm{ng} / \mathrm{ml}+/-77$ vs. HC $277 \mathrm{ng} / \mathrm{ml} \mathrm{+/-18.4,}$ $\mathrm{p}<0.0001)$ but there was no increase in NE activity in the same supernatants, nor any difference in released MPO activity compared to healthy controls.

Conclusions Our Results indicate that neutrophil phenotype is altered in $\mathrm{PAH}$, with a prolonged lifespan in response to a pro-inflammatory stimulus and increased release of NE. However, we did not detect a corresponding increase in NE activity, suggesting a concomitant increase in NE inhibitor release from PAH neutrophils. The potential role of this altered neutrophil phenotype in vascular remodelling requires further investigation.

\section{S112 CONDITIONED MEDIA FROM HUMAN PULMONARY ARTERIAL ENDOTHELIAL CELLS TREATED WITH HEPCIDIN OR HAEMOGLOBIN CAUSE PROLIFERATION AND MIGRATION OF HUMAN PULMONARY ARTERY SMOOTH MUSCLE CELLS}

T Shackshaft, SJ Wort, GJ Quinlan, L Ramakrishnan. Vascular Biology Group, NHLI, Imperial College London, London, UK

\subsection{6/thoraxjnl-2017-210983.118}

Introduction Pulmonary arterial hypertension (PAH) is characterised by vascular remodelling of pulmonary arterioles. Disrupted iron homeostasis including subclinical haemolysis are implicated in PAH, although exact mechanisms remain unknown. Interactions linked to altered iron handling consequent to release of mediators by human pulmonary artery endothelial cells (hPAECS) and/or human pulmonary artery smooth muscle cells (hPASMCs) may be important in this regard.

Objectives This study explored proliferative and migratory responses in hPASMCs linked to the primary exposure of hPAECs to indices of dysregulated iron homeostasis i.e., hepcidin or haemoglobin.

Methods hPAECs were challenged with haemoglobin (10 uM) or hepcidin (100-1000 ng/mL). Proliferation and migration of hPASMCs were analysed using xCELLigence RTCA instrument by measuring the changes in cell index/impedance.

Results Novel findings demonstrate that media conditioned with hepcidin for 48 hour from hPAECs increased proliferation of hPASMCs by $24 \%$ (95\% CI $0.256-47.9, \mathrm{p}<0.05)$ after 48 hour of treatment. Additionally, 24 hour conditioned media from both hepcidin and haemoglobin treated hPAECs caused 3.27 and 2.28 fold increases in migration respectively in hPASMCs.

Conclusion These findings highlight a direct role for variant iron homeostasis in hPAECs which is linked to subsequent functional responses in hPASMCs of importance to vascular remodelling. These studies may provide novel insights regarding mechanisms for haemoglobin and hepcidin driven proliferative and migratory responses of relevance to $\mathrm{PAH}$. 